\title{
The Radiating Unit Based on Hybrid Metal-Dielectric Structure with Bounded Sequence of Transverse Slots
}

\author{
Mayboroda D. V.*, Pogarsky S. A., Saprykin I. I. \\ Karazin Kharkov National University, 4, Svoboda sq., Kharkov, 61022, Ukraine
}

\begin{abstract}
The structure which consists of the five transverse slots based on inverted dielectric waveguide was experimentally investigated. The possibility of creating an antenna on the basis of the above mentioned waveguide with an acceptable radiation pattern and good agreement feed line and antenna are presented.
\end{abstract}

Keywords Inverted Dielectric Waveguide, Radiation, Slot

\section{Introduction}

The development of commun ications systems, telecomm unications and global positioning systems require a constant search for new technical solutions in the designing and creation of multifunctional devices of microwave and SHF bands. Also in telecommunications millimeter waves offer more bandwidth, and this technology is very promising especially for indoor cellular systems. Certainly, the same solutions for basic waveguides and resonators can be used also in the millimeter frequency range. However, there are many peculiarities specific for the millimeter-wave devices that call for more investigations necessary for development and perfection of the new technology.

Moreover, devices in this band must possess not only a high level of integral characteristics (the characteristics of the radiation, the possibility of excitation of wave modes with different polarization), but also easy to manufacture an acceptable tolerance, ease of excitation and concordance with external circuits.

Such a set of require ments significantly satis fy the hybrid metal-dielectric structures (HMDS) made on the basis of various modifications of dielectric waveguides [1-4]. A mong them, more and more attention is attracted by the so-called inverted dielectric waveguide (IDV), due to the fact that the IDV have some unique features not only in terms of physics of electromagnetic wave propagation, but also the structural features, in particular, the ability to create low-profile designs with branched topology.

To create a high-performance millimeter-wave radiating systems can be used HMDS with single (or sequences) slot discontinuities, one of the options is considered in the

* Corresponding author:

sergey.a.pogarsky@univer.kharkov.ua (Mayboroda D. V.)

Published online at http://journal.sapub.org/ijea

Copyright (C) 2012 Scientific \& Academic Publishing. All Rights Reserved paper.

\section{Basic Design}

As a basic construction the HDMS based on IDV was selected. Schematic representation of the radiating structure is shown in Figure 1. In this figure: 1 - seg ment of microstrip conductor, 2 - dielectric slab, 3-dielectric rod, 4-grounded base, 5 - slot discontinuity of rectangular shape. The elements of design had the following parameters: dielectric rod had dimensions $a \times b=6.8 \times 3 \mathrm{~mm}$ and was made of polystyrene with $\varepsilon_{1}=2.5$, the die lectric substrate was made of alumina $\varepsilon_{2}=9.6$, with dimensions $60 \times 48 \times 0.5 \mathrm{~mm}$, the length of microstrip had the width of $6 \mathrm{~mm}$. The axis of slot radiator with the size of $s \times w=4 \times 1.5 \mathrm{~mm}$ is oriented perpendicular to the axis of a dielectric rod (the direction of propagation of the eigenwave). Excitation was carried out by the method which is described in [4].

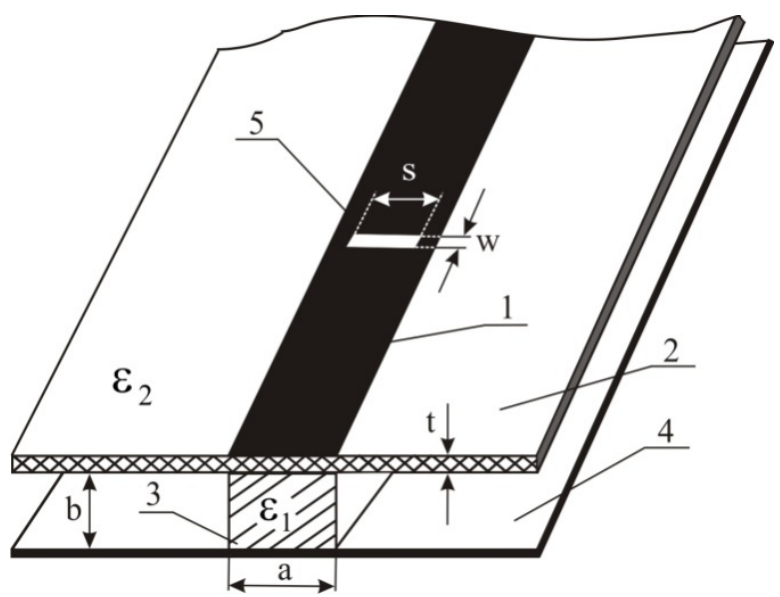

Figure 1. The schematic view of the structure

It is known that the effective radiation of a single-source and level of concordance with the external circuits may be estimated near frequency where minimum of magnitude of 
insertion losses and VSWR is observed. Figure 2 show the experimental curves, where 1 - attenuation, VSWR -2 .

As it is evident from the graphs, that near the frequency $f=29.45 \mathrm{GHz}$ the failure in the characteristic of attenuation is observed while the value of VSWR is less than the level of 1.5. Exactly the effective radiation from the structure may be pred icted near this frequency.

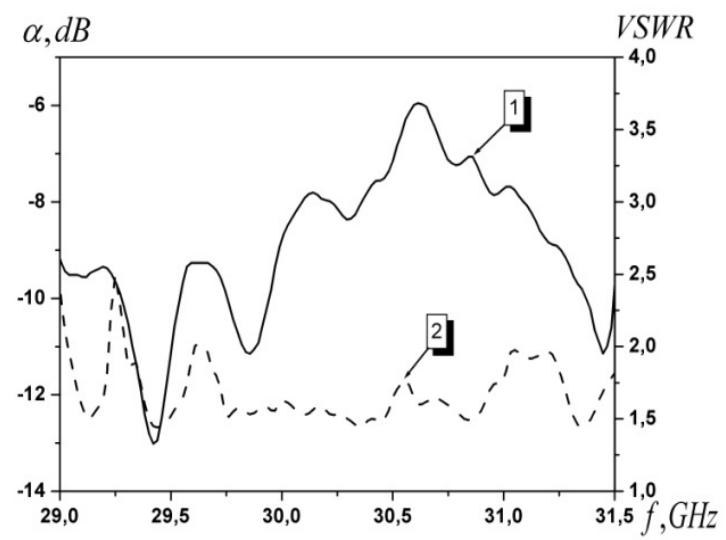

Figure 2. The attenuation and VSWR characteristic versus frequency $\left(\varepsilon_{1}=2.5, \varepsilon_{2}=9.6, t=1 \mathrm{~mm}, w=1.5 \mathrm{~mm}, s=4 \mathrm{~mm}, a=7 \mathrm{~mm}\right.$, $b=3 \mathrm{~mm})$

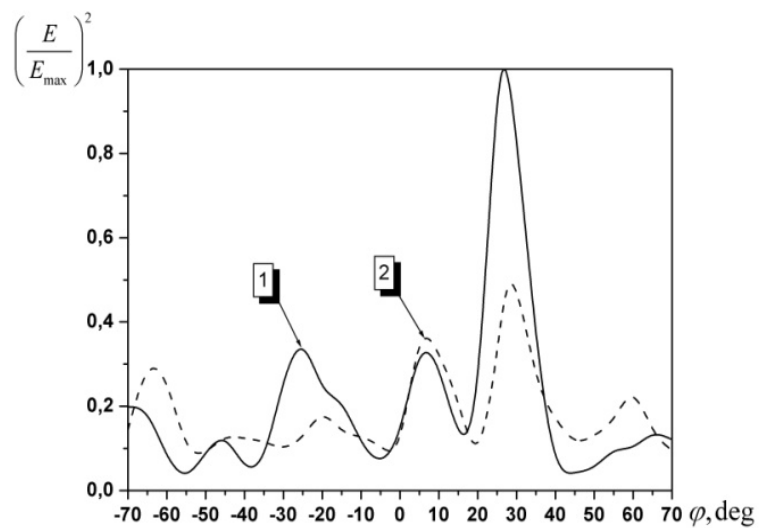

Figure 3. The normalized pattern characteristics $\left(\varepsilon_{1}=2.5, \varepsilon_{2}=9.6\right.$, $t=1 \mathrm{~mm}, w=1.5 \mathrm{~mm}, \mathrm{~s}=4 \mathrm{~mm}, a=7 \mathrm{~mm}, b=3 \mathrm{~mm})$

The experimental pattern characteristics are shown in Figure 3, which have been measured at two frequencies: the curve 1 corresponds to the frequency $f=29.45 \mathrm{GHz}$, the curve corresponds to the frequency $f=30.7 \mathrm{GHz}$. In both cases the shift of the maximum radiation fro $m$ the normal is observed, but in the resonance case, one can detect the main lobe clearly (in this case $s / \lambda_{g} \approx 1$, where $s$ is the length of the slot, $\lambda_{g}$ is the wavelength in HMDS). One can predict that reducing of spurious lobes may be achieved by increasing the quantity of radiators and optimizing the conditions of excitation of the structure.

\section{Radiating Unit}

Investigated basic design has been modified to improve electrodynamical performance. One of these modifications consists in use bounded periodical sequence of transverse rectangular slots in auxiliary microstrip conductor. And the second one consists in improving of excitation technique of basic structure (IDV). Schematic representation of the radiating structure is shown in Figure 4.

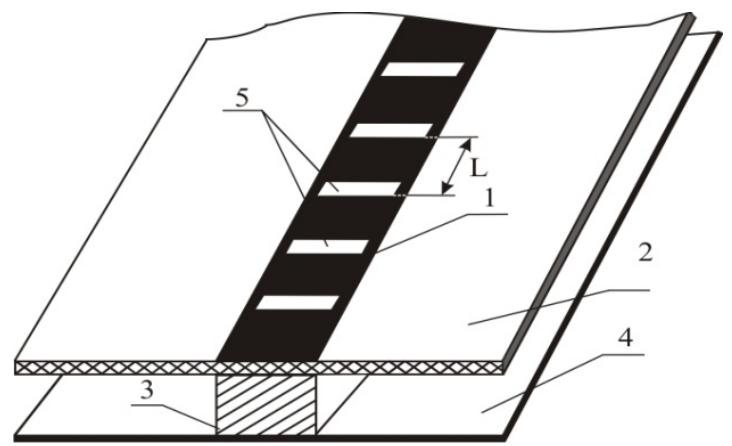

Figure 4. The schematic view of radiating unit

The principle difference between basic structure and modified radiating unit consists in presence of bounded periodical sequence of slot radiators (5). These radiators have such parameters: sizes $s \times w=4 \times 1.5 \mathrm{~mm}$, the distance between radiators were $L=6.5 \mathrm{~mm}$. This distance with taking into account wavelength shorting factor approximately equals to the wavelength in the structure. It is well known that effective radiation may be realized including under the condition of effective excitation of the basic structure (IDV). The best known method of excitation of such structures suggests that part of the dielectric rod is introduced into a rectangular waveguide. In this case the shape of the inputted into the waveguide part of rod and its length is very essential. These parameters will determine the level of insertion loss and the level of concordance with external circuits. Experimentally a lot of shapes of termination part of the rod have been investigated. In comparing with widely used shape in the form of ordinary scarf with Brewster's angle a long narrow side of the rod [5] the pyramidal and semi- pyramidal shapes are more attractive because they provide lowest level of attenuation and higher level of concordance with external circuits.

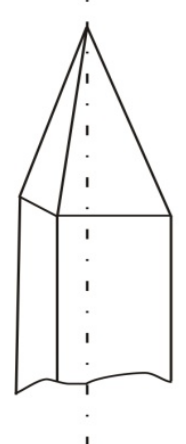

(a)

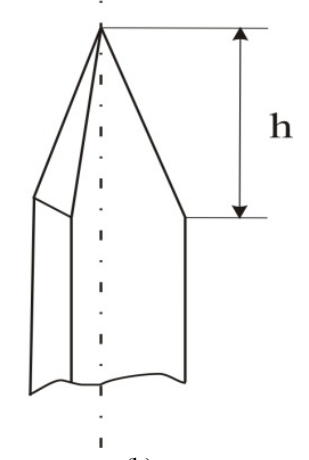

(b)
Figure 5. The shape of termination part of the rod

The shapes of terminal parts of the rod are presented in Figure 5 (a)-pyramidal, (b) - semi-pyramidal).

By experiments it has been established that the semi-pyramidalterminal part is more optimal. The parameter 
$h$ - the submerged length is optimal when $h \approx 1.5 \ldots 2 \lambda_{g}$, where $\lambda_{g}$ is wavelength in complicated structure.

In Figure 6 the attenuation and VSWR characteristics versus frequency are presented. The curve 1 reflects the dependence of magnitude attenuation, curve 2 shows the dependence of the level of VSWR versus frequency. As it is evident fro $m$ the figures the curve 2 have a lot of oscillations, which is caused by the influence of slot discontinuities on the wave propagating in the channel, but in certain frequency band $(28-30 \mathrm{GHz})$ it is observed frequencies within which the VSWR is about the level of $1.75 \ldots 1.85$. It may be predicted that in this band it is possible to obtain effective radiation from the structure.

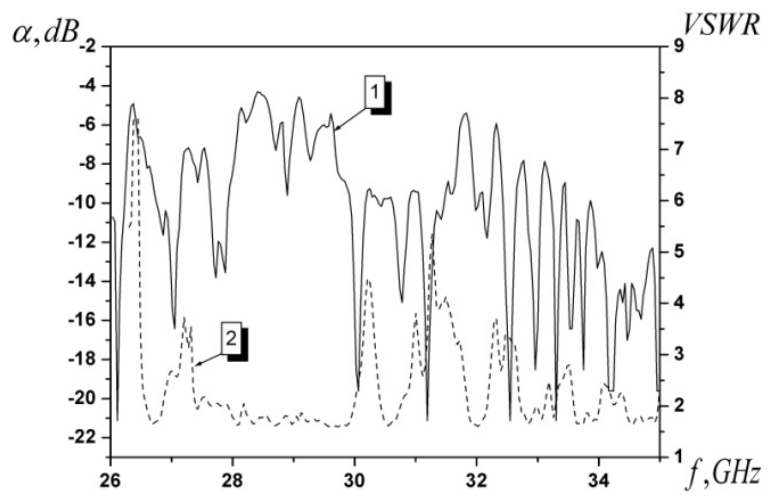

Figure 6. The attenuation and VSWR characteristics versus frequency $\left(\varepsilon_{1}=2.5, \varepsilon_{2}=9.6, t=1 \mathrm{~mm}, w=1.5 \mathrm{~mm}, s=4 \mathrm{~mm}, a=7 \mathrm{~mm}\right.$, $b=3 \mathrm{~mm}, L=6.5 \mathrm{~mm})$

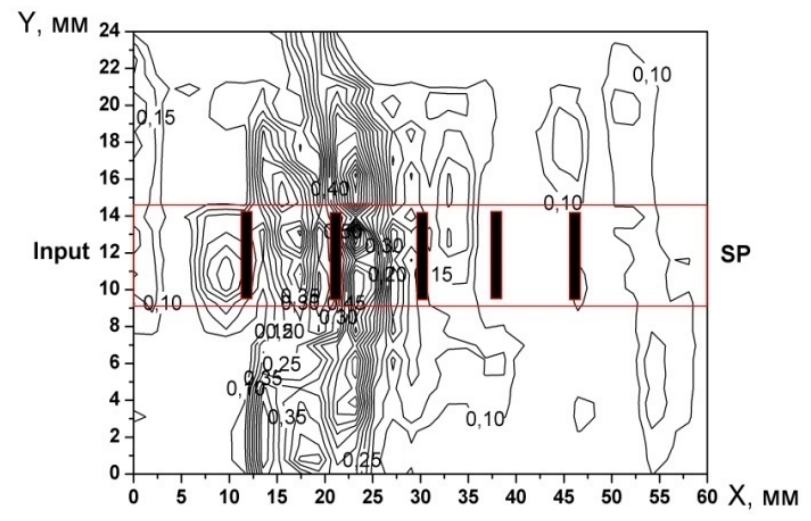

Figure 7. The near field structure at $30.18 \mathrm{GHz}\left(\varepsilon_{1}=2.5, \varepsilon_{2}=9.6\right.$, $t=1 \mathrm{~mm}, w=1.5 \mathrm{~mm}, \mathrm{~s}=4 \mathrm{~mm}, a=7 \mathrm{~mm}, b=3 \mathrm{~mm}, L=6.5 \mathrm{~mm})$

For a more detailed investigation of the nature of the interaction mechanism between the waveguiding system and the radiator the experiments to determine the structure of the electric field in the near zone have been carried out. In Figure 7 the electric field in contour form of the near field at a frequency $30.18 \mathrm{GHz}$ have shown. This characteristic measured by the method of moving probe. It is necessary to mention that the amplitude distribution mode between radiators is very important characteristic because it defines the power part at every radiator and in turn it defines the pattern characteristic.
As it can be seen from the figure, the maximum of electric field is localized near the first three slot radiators. Obviously, we can predict that the most of the radiated power may be detected near these radiators. The non-equidistant arrangement of the radiators may be proposed for a more uniform distribution of input power to radiators.

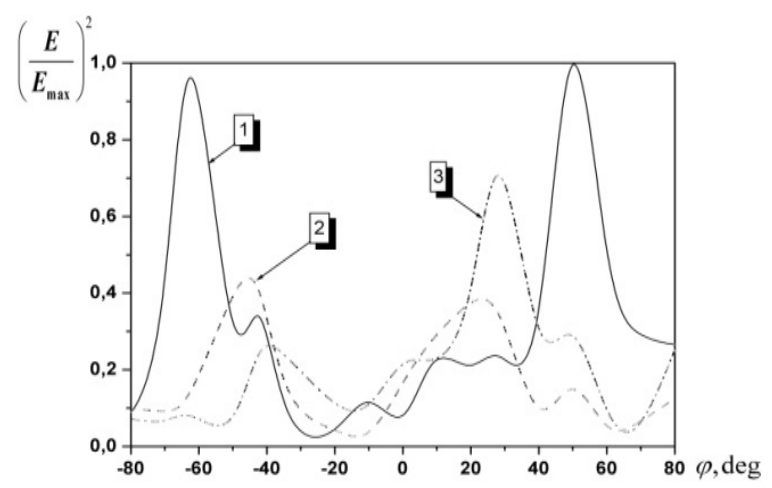

Figure 8. The pattern characteristics of the structure at different frequencies $\left(\varepsilon_{1}=2.5, \varepsilon_{2}=9.6, t=1 \mathrm{~mm}, w=1.5 \mathrm{~mm}, \mathrm{~s}=4 \mathrm{~mm}\right.$, $a=7 \mathrm{~mm}, b=3 \mathrm{~mm}, L=6.5 \mathrm{~mm})$

The experimentally radiation pattern characteristics of the structure at different frequencies are shown in Figure 8, where curve 1 corresponds to the frequency of $30.19 \mathrm{GHz}, 2$ - frequency of $31.44 \mathrm{GHz}$ and 3 - the frequency of $33.4 \mathrm{GHz}$. All diagrams are normalized by global maximum. As follows from the dependencies, this structure allows to realize the maximum radiation as in a single beam (curve 3 ), and within the two beams (curve 1).

\section{Conclusions}

Thus, the carried out experimental researches show that the using of an alternative transmission line, for example inverted dielectric waveguide, allows to design thefunctiona 1 elements with high level of integral characteristics.

\section{REFERENCES}

[1] Zong-Wen Li. A $35 \mathrm{GHz}$ low side-lobe leaky-wave antenna with periodic metallic grating structures of nonuniform width on the dielectric waveguide // Microwave Conference Proceedings Asia-Pacific APMC 97 (Hong-Kong Dec. 2-5, 1997). H-K., Vol.2. 1997. P. 641 - 644.

[2] Zong-Wen Li. A $61 \mathrm{GHz}$ Doppler radar using inverted strip dielectric waveguide // Microwave Symposium Digest MWSYM 1992 (New Mexico Jun. 1-5, 1992). N-M., 1992. P. 629-632.

[3] D. Mayboroda, S. Pogarsky, I. Saprikin. The guiding properties of strip dielectric waveguides and possibility of functional and radiating elements designing // Joint 29th International Conference on Infrared and Millimeter Waves and 12th International Conference on Terahertz Electronics, 2004 (27 Sept.-1 Oct. 2004). 2004. P. 701-702. 
[4] D. Mayboroda, S. Pogarsky, S. Pshenichnaya, I.Saprikin. The radiator of Ku-band based on inverted dielectric wave-guide // 5th International Conference on Ultra-wide band and Ultrashort Impulse Signals UWBUSIS 2010 (Sevastopol, September 10-13. 2010). Sevastopol, 2010. P. 215-217.
[5] Itoh T. Passive Millimeter-Wave IC Components Made of Inverted Strip Dielectric Waveguides // International Microwave Symposium IEEE-MWSYM 1976 (New Jersey, October 14-16, 1976). NJ., 1976. P. 182-184. 